\title{
QUANTIZATION OF THE STRING INSPIRED DILATON GRAVITY AND THE BIRKHOFF THEOREM
}

\author{
Marco Cavaglià ${ }^{(a)},{\text { Vittorio de } \text { Alfaro }^{(b, d)} \text { and Alexandre T. Filippov }}^{(c)}$ \\ (a) Max-Planck-Institut für Gravitationsphysik, \\ Albert-Einstein-Institut, \\ Schlaatzweg 1, D-14473 Potsdam, Germany. \\ (b) Dipartimento di Fisica Teorica dell'Università di Torino, \\ Via Giuria 1, I-10125 Torino, Italy. \\ (c) Joint Institute for Nuclear Research \\ R-141980 Dubna, Moscow Region, Russia. \\ (d) INFN, Sezione di Torino, Italy.
}

\begin{abstract}
We develop a simple scheme of quantization for the dilaton CGHS model without scalar fields, that uses the Gupta-Bleuler approach for the string fields. This is possible because the constraints can be linearized classically, due to positivity conditions that are present in the model (and not in the general string case). There is no ambiguity nor anomalies in the quantization. The expectation values of the metric and dilaton fields obey the classical requirements, thus exhibiting at the quantum level the Birkhoff theorem.
\end{abstract}

PAC(S): 04.60.-m, 04.60.Kz, 03.70.+k.

Keyword(s): Quantum Gravity, Two-Dimensional models, Canonical Quantization, Field Theory

E-Mail: cavaglia@aei-potsdam.mpg.de

E-Mail:vda@to.infn.it

E-Mail: filippov@thsun1.jinr.dubna.su 


\section{Introduction.}

Recently, much work has been done on gravitational models in $1+1$ dimensions, the solution of which may hopefully clarify some difficult problems of quantum black holes and, more generally, of quantum gravity in higher dimensions [1]. Most of the models studied in some detail in the literature are special cases of the general dilaton gravity coupled to gauge and scalar fields (for a very compact overview and references see [2]). In general, these models are not integrable and their solution cannot be found even classically. However, interesting models which are classically integrable are also known whose solutions can be explicitly written in terms of free massless fields (the most general class of such models is presented in [2]). Unfortunately, not much is known about their quantization: only the so-called CGHS model [3] has been studied in detail (see Refs. $[4,5,6]$; for a review of earlier results see also [1]). These investigations revealed two main obstacles to quantization: first, the quantum canonical transformations to free fields are highly nontrivial and difficult to construct; second, the quantization is obstructed by anomalies in the commutator of the constraints, and the theory needs to be modified. In the CGHS model, the canonical transformations and modification of the constraints have recently been implemented $[5,6]$. However, the quantization of more general integrable models is a completely open problem.

The problems mentioned persist even in the dilaton gravity models not coupled to scalar matter fields. The general dilaton gravity model is $[7,8,2]$

$$
\mathcal{L}=\sqrt{-g}\left[U(\varphi) R(g)+V(\varphi)+W(\varphi) g^{\mu \nu} \partial_{\mu} \varphi \partial_{\nu} \varphi\right]
$$

where $\mu, \nu=0,1 ; U, V, W$ may be arbitrary functions of $\varphi$, and $R$ is the scalar curvature. Locally, we may always choose $U=\varphi$ (or, $U=\exp (c \varphi)$, etc.). Using the Weyl rescaling, $g_{\mu \nu}=\Omega(\varphi) \bar{g}_{\mu \nu}$, we may make $W$ equal to any given function (e.g. $\bar{W}=0)$. The CGHS model without matter is equivalent, in this sense, to the theory with $W=0$ and $V=$ const (with $V=\varphi$ we get the Jackiw-Teitelboim model).

The general dilaton gravity described by Eq. (1) is integrable and both the metric and dilaton fields may be expressed in terms of one free (D'Alembert) field and of one invariant parameter which is a local integral of motion independent of the coordinates. (For the Schwarzschild black hole, it is the black hole mass.) Using the gauge in which this free field is one of the coordinates, one finds that all solutions depend on one coordinate. This means that the metric can be explicitly expressed in terms of the dilaton and thus has at least one horizon. (For more details see, e.g. Ref. [2].) These properties constitute a generalization of the classical Birkhoff theorem for spherically symmetric gravity in any dimension (we thus call, somewhat improperly, "static" these solutions).

The drastic reduction of the dilaton gravity field theory to a finite-dimensional

dynamical system signals that the general dilaton gravity is actually a topological 
theory. ${ }^{1}$ The Birkhoff theorem is also valid for the dilaton gravity (1) coupled to Abelian gauge fields $([8,2])$. However, any coupling to scalar fields destroys the topological nature of the theory and invalidates the Birkhoff theorem. Moreover, $0+1$ dimensional solutions of dilaton gravity coupled to scalar matter have no horizons (the "no horizon theorem" [2]). As a consequence, the coupling to scalar fields cannot be treated perturbatively, even in the classical theory.

Unlike the theories coupled to scalars, the general dilaton gravity model can be quantized by first reducing it to a dynamical system with a single constraint. (This may be regarded as a particular gauge fixing.) The quantization of the finitedimensional system so obtained is more or less straightforward, and the resulting Hilbert space is spanned by the eigenvectors of the (gauge invariant) mass operator. This quantization approach, that uses first the Birkhoff theorem and then the quantization algorithm, has been introduced in Ref. [11] in the case of the $3+1$ dimensional Schwarzschild black hole; the procedure can be generalized to any pure two dimensional dilaton gravity model. ${ }^{2}$

A further approach to the quantization of the pure dilaton gravity models does not use the topological nature of the models nor the Birkhoff theorem. Since the classical solution can be written as a function of a single free field, one may try to find a canonical transformation of dilaton gravity to constrained free fields. This transformation is known for some time for the CGHS case [5,6], and recently an interesting new proposal in this direction has been advanced [12]. However, up to now an explicit canonical transformation has not been constructed for the general case and this approach was worked out only for the CGHS model which was the subject of deep investigations (with and without scalar matter, see Refs. $[4,5,6]$ ).

The main results of these investigations are clearly summarized in the report [13]. Although a beautiful canonical transformation which linearizes the CGHS model and represents it in terms of an infinite bosonic string does exist, the quantization is not straightforward even in the absence of scalar fields. Indeed, due to the presence of anomalies, different quantum theories corresponding to the same classical model can be found. In particular, in the Schrödinger representation there exists a quantization scheme in which the anomaly in the pure dilaton gravity is cancelled [5]. However, this scheme is based on the use of negative energy states for the string fields instead of the standard string quantization that introduces negative norm states, and the result obtained has no evident connection to the quantum version of the reduced theory via the Birkhoff theorem.

1 The topological nature of the CGHS and Jackiw-Teitelboim models is well-known: they are topological BF theories (see $[4,9,5]$ ). For the topological formulation of the general dilaton gravity see [10] and references therein.

2 It is not difficult to rewrite all the formulas of Ref. [11] for the simpler CGHS case. The treatment of more general potentials $V(\varphi)$, when many horizons may exist, requires a more careful consideration. 
In this paper we consider a further scheme for the pure dilaton CGHS model, that directly proves the Birkhoff reduction at the quantum level and does not produce any anomalies. The quantization is carried out, by use of the standard Gupta-Bleuler method, on the string variables. This is possible because the model has actually more information in it than the canonically transformed version described by a couple of string fields. In fact, we will argue that this model is not equivalent to a string theory.

In the first place, there exists a gauge invariant local mass that cannot be expressed purely in terms of the string fields. Moreover, further information comes from the canonical transformation between the original fields and the string ones, under the form of positivity conditions for certain functions. This allows an essential step: the linearization of the constraints. Let us stress that the linearized constraints generate reparametrizations of the metric. There is no need to consider the original quadratic constraints as fundamental operators.

Hence, it becomes possible to quantize the model with the standard choice of the quantum vacuum by the Gupta-Bleuler method. There is no anomaly in the algebra of the linearized constraints (the algebra of the quadratic constraints does not concern us any more). This quantization of the string-like fields is essentially

equivalent to the "Schrödinger" quantization that uses positive norms and negative energy states [5].

The only gauge invariant operators are the mass and its conjugate momentum (conjugate operators were discussed in Refs. [14,8]). The ground state of the string must be labeled by the eigenvalue of the mass operator (as discussed in a different context in Refs. $[4,8])$.

Our quantization procedure explicitly shows how the reduction to quantum mechanics is achieved by the Gupta-Bleuler quantization of the field theory. With linearized constraints, the quantized field theory has the same content as scalarlongitudinal electrodynamics and is pure gauge. The set of Gupta-Bleuler states corresponds to reparametrization of the coordinates, expectation values of the metric and dilaton fields agree with the corresponding classical quantities and the "Birkhoff reduced" quantum theory is recovered.

\section{Action and Hamiltonian Formalism.}

Our starting point is the two-dimensional action related by a Weyl transformation to the pure dilaton CGHS [3] model

$$
S=\int d^{2} x \sqrt{-g}\left[\varphi R-\frac{\lambda}{2}\right],
$$

here $g_{\mu \nu}$ is a two-dimensional metric and $\varphi$ is the dilaton field (for $R$ we follow the conventions of [15]). As in [5] we write the two-dimensional metric as

$$
g_{\mu \nu}=\rho\left(\begin{array}{cc}
\alpha^{2}-\beta^{2} & \beta \\
\beta & -1
\end{array}\right) \text {. }
$$


Here $\alpha\left(x_{0}, x_{1}\right)$ and $\beta\left(x_{0}, x_{1}\right)$ play the role of the lapse function and of the shift vector respectively; $\rho\left(x_{0}, x_{1}\right)$ represents the dynamical gravitational degree of freedom. The coordinates $x_{0}, x_{1}$ are both defined on $\mathbb{R}$. It is convenient to introduce the variable $f=\ln \rho$. Using (3) the action (2) can be written in the Hamiltonian form (see e.g. $[5]) \mathrm{as}^{3}$

$$
S=\int d^{2} x\left[\dot{f} \pi_{f}+\dot{\varphi} \pi_{\varphi}-\alpha \mathcal{H}-\beta \mathcal{P}\right],
$$

where $\pi_{f}$ and $\pi_{\varphi}$ are the conjugate momenta to $f$ and $\varphi$ respectively, and $\mathcal{H}$ and $\mathcal{P}$ are the constrained super-Hamiltonian and super-momentum:

$$
\begin{aligned}
& \mathcal{H}=\pi_{f} \pi_{\varphi}+f^{\prime} \varphi^{\prime}-2 \varphi^{\prime \prime}+\frac{\lambda}{2} e^{f}=0, \\
& \mathcal{P}=2 \pi_{f}^{\prime}-\pi_{\varphi} \varphi^{\prime}-\pi_{f} f^{\prime}=0 .
\end{aligned}
$$

We may also define a functional $M$ of the canonical variables which is conserved under time and space translations (analogous to the Schwarzschild mass) $[8,2,16]$. In our notations $M$ is given by

$$
M=\frac{\lambda}{2} \varphi+e^{-f}\left(\pi_{f}^{2}-\varphi^{\prime 2}\right) .
$$

It is straightforward to prove that $\dot{M}=M^{\prime}=0$ using the equations of motion and the constraints.

The Birkhoff reduction to static configuration (depending on one coordinate) may be stated as follows. We may set $\alpha=1$ and $\beta=0$ and introduce the coordinates

$$
u=\frac{1}{2}\left(x_{0}+x_{1}\right), \quad v=\frac{1}{2}\left(x_{0}-x_{1}\right) ;
$$

the two-dimensional line element corresponding to the metric tensor (3) becomes

$$
d s^{2}=4 \rho(u, v) d u d v .
$$

A metric of this form is static if and only if $\rho$ can be cast in the form [2]

$$
\rho(u, v)=h(\Psi) \frac{d a(u)}{d u} \frac{d b(v)}{d v}, \quad \Psi \equiv a(u)+b(v),
$$

where $a$ and $b$ are arbitrary functions. This metric depends on one coordinate. If, in addition, $\varphi$ depends only on $\Psi$, the solution will be called static. To see that all the solutions of (2) are static we write the constraints and the equations of motion in the coordinates (7) [2]

$$
\begin{aligned}
& \partial_{u}\left(e^{-f} \partial_{u} \varphi\right)=\partial_{v}\left(e^{-f} \partial_{v} \varphi\right)=0, \\
& \partial_{u} \partial_{v} f=0, \quad \partial_{u} \partial_{v} \varphi+\frac{\lambda}{2} e^{f}=0,
\end{aligned}
$$

3 Note that all the formulae of this section can be easily rewritten for the general dilaton gravity (1). 
where the constraints (10a) are valid for the generic model (1) (but will be destroyed by adding any coupling to additional scalar fields). The constraints (10a) can be solved in terms of a free field:

$$
\begin{aligned}
& \rho \equiv e^{f}=\frac{d F}{d \Psi} \partial_{u} \Psi \partial_{v} \Psi, \\
& \varphi=F(\Psi), \\
& \partial_{u} \partial_{v} \Psi=0 .
\end{aligned}
$$

This shows that all the solutions of the generic dilaton gravity (1) are static, which is the generalized Birkhoff theorem. The function $F(\Psi)$ is determined by the equations of motion and depends on the potential $V(\varphi)$. For the CGHS model we find $F(\Psi)$ from $(10 b)$

$$
F(\Psi)=C_{0} e^{-\lambda \Psi / 2}+\frac{2 M}{\lambda} .
$$

Here $C_{0}$ is an integration constant; the second integration constant was expressed in terms of the constant $M$ by using (6). One sees that $M$ appears as a zero mode of the field $\varphi$. Now let us recall the canonical free field formalism that will be the starting point for the quantum theory.

\section{String variables and linearization of the constraints.}

Let us use the transformation [5]

$$
\begin{aligned}
& A_{0}=\frac{2}{\lambda} e^{-f / 2}\left(\pi_{f} \cosh \Sigma-\varphi^{\prime} \sinh \Sigma\right), \pi^{0}=-\lambda e^{f / 2} \cosh \Sigma-\lambda A_{1}^{\prime}, \\
& A_{1}=\frac{2}{\lambda} e^{-f / 2}\left(\pi_{f} \sinh \Sigma-\varphi^{\prime} \cosh \Sigma\right), \quad \pi^{1}=\lambda e^{f / 2} \sinh \Sigma+\lambda A_{0}^{\prime},
\end{aligned}
$$

where

$$
\Sigma\left(x_{1}\right)=\frac{1}{2} \int_{-\infty}^{x_{1}} d x_{1}^{\prime} \pi_{\varphi}\left(x_{1}^{\prime}\right)
$$

The above transformation is canonical for the field variables $\left(-\varphi^{\prime}, \Sigma, f, \pi_{f} ; A_{\alpha}, \pi_{\alpha}\right)$. Note that the inverse transformation only defines $\varphi$ up to a zero mode $\varphi_{0}\left(x_{0}\right) \equiv$ $\varphi\left(x_{0}, c\right)$. To make the transformation (12) invertible, one has to supplement the new field variables $A_{\alpha}, \pi_{\alpha}$ by a pair of conjugate variables, e.g. $\varphi\left(x_{0}, c=\infty\right)$ and $2 \Sigma(\infty)$ $\left(\varphi_{0}\right.$ will commute with all the field variables if we choose $\left.c=\infty\right)$.

Using (12) the two constraints become

$$
\begin{aligned}
\mathcal{H} & =\frac{1}{2 \lambda} \pi^{\alpha} \pi_{\alpha}+\frac{\lambda}{2} A^{\prime \alpha} A_{\alpha}^{\prime}=0, \\
\mathcal{P} & =-\pi^{\alpha} A_{\alpha}^{\prime}=0 .
\end{aligned}
$$

Let us introduce the metric and the Levi - Civita tensors as

$$
\eta^{\alpha \beta}=\left(\begin{array}{cc}
1 & 0 \\
0 & -1
\end{array}\right) ; \quad \varepsilon^{\alpha \beta}=\left(\begin{array}{cc}
0 & 1 \\
-1 & 0
\end{array}\right) .
$$


The functional $M$ defined in (6) is represented as

$$
M=M_{0}+\frac{\lambda}{4} \int_{b}^{x_{1}} d x_{1}^{\prime}\left(\varepsilon^{\alpha \beta} \pi_{\alpha} A_{\beta}+\lambda A^{\alpha} A_{\alpha}^{\prime}\right) .
$$

(The value of $b$ is irrelevant.) Here $M_{0}$ must be a constant since $M$ is independent of $t$. The zero mode $\varphi_{0}$ and the zero modes of the string fields can be related to $M_{0}$ but we do not need their precise relationship (see [6]). On the equations of motion we have $M=M_{0}$. Let us introduce the operators $\mathcal{C}^{\alpha}$ and $\mathcal{D}^{\alpha}$

$$
\begin{aligned}
& \mathcal{C}^{\alpha}=\pi^{\alpha}-\lambda \varepsilon^{\alpha \beta} A_{\beta}^{\prime}, \\
& \mathcal{D}^{\alpha}=\pi^{\alpha}+\lambda \varepsilon^{\alpha \beta} A_{\beta}^{\prime} .
\end{aligned}
$$

$\dot{M}$ and $M^{\prime}$ are proportional to $\mathcal{C}^{\alpha}$. The classical quantities $\mathcal{H}, \mathcal{P}, M, \rho, \varphi$ can be written as functions of $\mathcal{C}^{\alpha}, \mathcal{D}^{\alpha}$ and $A_{\alpha}$. They read

$$
\begin{aligned}
\mathcal{H} & =\frac{1}{2 \lambda} \mathcal{C}^{\alpha} \mathcal{D}_{\alpha}, \\
\mathcal{P} & =\frac{1}{2 \lambda} \varepsilon_{\alpha \beta} \mathcal{D}^{\alpha} \mathcal{C}^{\beta}, \\
M & =M_{0}-\frac{\lambda}{4} \int_{b}^{x_{1}} d x_{1}^{\prime} \varepsilon^{\alpha \beta} A_{\alpha} \mathcal{C}_{\beta}, \\
\rho & =\frac{1}{\lambda^{2}} \mathcal{D}^{\alpha} \mathcal{D}_{\alpha}, \\
\varphi & =\frac{2 M}{\lambda}-\frac{\lambda}{2} A_{\alpha} A^{\alpha} .
\end{aligned}
$$

The Poisson brackets of $\mathcal{C}^{\alpha}$ and $\mathcal{D}^{\alpha}$ are

$$
\left[\mathcal{C}^{\alpha}\left(x_{0}, x\right), \mathcal{D}^{\beta}\left(x_{0}, y\right)\right]=2 \lambda \varepsilon^{\alpha \beta} \partial_{y} \delta(x-y) .
$$

Now we show that the constraints can be linearized, using a suitable redefinition of the Lagrange multipliers. The linearized constraints are the functions $\mathcal{C}^{\alpha}$. Thus in this form of the theory the generators are linear in the string variables $A_{\alpha}$.

It is useful to introduce the conjugate variables $[6]\left(\mathcal{P}_{ \pm}, \mathcal{X}_{ \pm}\right)$where

$$
\begin{array}{ll}
\mathcal{P}_{-}=\frac{1}{2 \sqrt{\lambda}}\left(\mathcal{C}^{0}-\mathcal{C}^{1}\right), & \mathcal{X}_{-}^{\prime}=-\frac{1}{2 \sqrt{\lambda}}\left(\mathcal{D}^{0}+\mathcal{D}^{1}\right), \\
\mathcal{P}_{+}=\frac{1}{2 \sqrt{\lambda}}\left(\mathcal{C}^{0}+\mathcal{C}^{1}\right), & \mathcal{X}_{+}^{\prime}=\frac{1}{2 \sqrt{\lambda}}\left(\mathcal{D}^{0}-\mathcal{D}^{1}\right),
\end{array}
$$

and also define the constraints $\mathcal{H}_{+}$and $\mathcal{H}_{-}$that generate reparametrization in $u$ and $v$; they can be written as functions of the constraints $\mathcal{P}_{ \pm}$and of the space derivative of the conjugate constraints, $\mathcal{X}_{ \pm}^{\prime}$ :

$$
\begin{aligned}
& \mathcal{H}_{+} \equiv \mathcal{H}+\mathcal{P}=\frac{1}{2 \lambda}\left(\mathcal{D}^{0}+\mathcal{D}^{1}\right)\left(\mathcal{C}^{0}-\mathcal{C}^{1}\right)=-2 \mathcal{X}_{-}^{\prime} \mathcal{P}_{-}, \\
& \mathcal{H}_{-} \equiv \mathcal{H}-\mathcal{P}=\frac{1}{2 \lambda}\left(\mathcal{D}^{0}-\mathcal{D}^{1}\right)\left(\mathcal{C}^{0}+\mathcal{C}^{1}\right)=2 \mathcal{X}_{+}^{\prime} \mathcal{P}_{+}
\end{aligned}
$$


Using these variables the Lagrangian density reads

$$
\mathcal{L}=\dot{\mathcal{X}}_{+} \mathcal{P}_{+}+\dot{\mathcal{X}}_{-} \mathcal{P}_{-}-l_{+} \mathcal{H}_{+}-l_{-} \mathcal{H}_{-}
$$

where $l_{ \pm}$are the suitable combinations of the Lagrange multipliers. In Appendix we show that $\mathcal{H}=0$ and $\mathcal{P}=0$ are satisfied iff $\mathcal{C}^{\alpha}=0$ since $\mathcal{X}^{\prime}{ }_{ \pm}$have definite signs. Accordingly, the coefficients $\mathcal{X}_{ \pm}$can be reabsorbed in the Lagrange multipliers. We redefine the multipliers as

$$
r_{+}=2 \mathcal{X}_{+}^{\prime} l_{-}, \quad r_{-}=-2 \mathcal{X}_{-}^{\prime} l_{+}
$$

The Lagrangian density is now

$$
\mathcal{L}=\dot{\mathcal{X}}_{+} \mathcal{P}_{+}+\dot{\mathcal{X}}_{-} \mathcal{P}_{-}-r_{+} \mathcal{P}_{+}-r_{-} \mathcal{P}_{-}
$$

The linear constraints, $\mathcal{P}_{ \pm}$or $\mathcal{C}_{\alpha}$, generate the reparametrizations of the metric. Indeed we have the Poisson brackets

$$
\left[\rho\left(x_{0}, x\right), \mathcal{C}^{\alpha}\left(x_{0}, y\right)\right]=4 \varepsilon^{\alpha \beta} \lambda^{-1} \mathcal{D}_{\beta}\left(x_{0}, x\right) \partial_{y} \delta(x-y)
$$

As a consequence of the linearization, the theory can be quantized in a simple scheme. Before doing that, let us see the form that the classical solution (11) takes in terms of the fields $A_{\alpha}$. The equations of motion are

$$
\lambda \dot{A}_{\alpha}=\eta_{\alpha \beta} \pi^{\beta}, \quad \dot{\pi}^{\alpha}=\lambda \eta^{\alpha \beta} A_{\beta}^{\prime \prime}
$$

and the solution is

$$
A_{\alpha}=U_{\alpha}(u)+V_{\alpha}(v) .
$$

The linear constraints $\mathcal{C}^{\alpha}=0$ correspond to

$$
\eta^{\alpha \beta} \partial_{\alpha} A_{\beta}=0, \quad \varepsilon^{\alpha \beta} \partial_{\alpha} A_{\beta}=0, \quad \text { or }, \quad U_{0}(u)=U_{1}(u), \quad V_{0}(v)=-V_{1}(v) .
$$

It is easy to prove that the solution $(29,30)$ coincides with (11). Substituting Eqs. $(29,30)$ into $(18 d, e)$ one obtains

$$
\begin{aligned}
& \rho \equiv e^{f}=4 \frac{d U_{0}(u)}{d u} \frac{d V_{0}(v)}{d v}, \\
& \varphi=\frac{2 M}{\lambda}-2 \lambda U_{0}(u) V_{0}(v) .
\end{aligned}
$$

This solution coincides with (11) if $\Psi$ is defined by

$$
C_{0} e^{-\lambda \Psi / 2}=-2 \lambda U_{0}(u) V_{0}(v) .
$$

The "staticity" of the classical solution is thus embodied in Eqs. (31). 


\section{Quantization.}

The quantization starts from the introduction of the Lagrangian ${ }^{4}$

$$
\mathcal{L}=\frac{1}{2} \partial_{\mu} A_{\alpha} \partial_{\nu} A_{\beta} \eta^{\mu \nu} \eta^{\alpha \beta}
$$

The commutation relations are

$$
\left[A_{\alpha}(x), A_{\beta}(y)\right]=-\eta_{\alpha \beta} \int \frac{d^{2} k}{2 \pi} \delta\left(k^{2}\right) \varepsilon\left(k_{0}\right) e^{i k(x-y)} .
$$

The field expansion is

$$
A_{\alpha}=\int_{-\infty}^{\infty} \frac{d k}{2 \sqrt{\pi \omega}}\left\{b_{\alpha}(k) e^{-i \omega x_{0}+i k x_{1}}+b_{\alpha}^{\dagger}(k) e^{i \omega x_{0}-i k x_{1}}\right\},
$$

where $\omega=|k|$. From (29) we obtain

$$
\begin{aligned}
& U_{\alpha}=\int_{0}^{\infty} \frac{d k}{2 \sqrt{\pi k}}\left\{a_{\alpha}(k) e^{-2 i k u}+a_{\alpha}^{\dagger}(k) e^{2 i k u}\right\} \\
& V_{\alpha}=\int_{0}^{\infty} \frac{d k}{2 \sqrt{\pi k}}\left\{b_{\alpha}(k) e^{-2 i k v}+b_{\alpha}^{\dagger}(k) e^{2 i k v}\right\}
\end{aligned}
$$

and $a_{\alpha}(k)=b_{\alpha}(-k), k>0$. Consequently $(k>0)$ the non-vanishing commutators are

$$
\left[a_{\alpha}(k), a_{\beta}^{\dagger}\left(k^{\prime}\right)\right]=\eta_{\alpha \beta} \delta\left(k-k^{\prime}\right), \quad\left[b_{\alpha}(k), b_{\beta}^{\dagger}\left(k^{\prime}\right)\right]=\eta_{\alpha \beta} \delta\left(k-k^{\prime}\right) .
$$

It follows that

$$
\left[U_{\alpha}\left(u_{1}\right), U_{\beta}\left(u_{2}\right)\right]=-\frac{i}{4} \eta_{\alpha \beta} \varepsilon\left(u_{1}-u_{2}\right), \quad\left[V_{\alpha}\left(v_{1}\right), V_{\beta}\left(v_{2}\right)\right]=-\frac{i}{4} \eta_{\alpha \beta} \varepsilon\left(v_{1}-v_{2}\right) .
$$

The canonical quantities $\left(\mathcal{P}_{ \pm}, \mathcal{X}_{ \pm}\right)$can be expressed in function of $q_{a, b}(k)$ and $p_{a, b}(k)$ where

$$
\begin{array}{ll}
q_{a}=a_{0}-a_{1}, & q_{b}=b_{0}+b_{1}, \\
p_{a}=a_{0}+a_{1}, & p_{b}=b_{0}-b_{1} .
\end{array}
$$

Their non vanishing commutators are

$$
\left[q_{a}(k), p_{a}^{\dagger}\left(k^{\prime}\right)\right]=\left[q_{b}(k), p_{b}^{\dagger}\left(k^{\prime}\right)\right]=2 \delta\left(k-k^{\prime}\right)
$$

\footnotetext{
4 From now on we set $\lambda=1$. There is no real loss of generality while the formulae become more elegant.
} 
We have

$$
\begin{aligned}
& \mathcal{P}_{+}=-i \int_{0}^{\infty} \frac{d k k}{2 \sqrt{\pi k}}\left(q_{a}(k) e^{-2 i k u}-q_{a}^{\dagger}(k) e^{2 i k u}\right), \\
& \mathcal{P}_{-}=-i \int_{0}^{\infty} \frac{d k k}{2 \sqrt{\pi k}}\left(q_{b}(k) e^{-2 i k v}-q_{b}^{\dagger}(k) e^{2 i k v}\right), \\
& \mathcal{X}_{+}=\int_{0}^{\infty} \frac{d k}{2 \sqrt{\pi k}}\left(p_{a}(k) e^{-2 i k u}+p_{a}^{\dagger}(k) e^{2 i k u}\right), \\
& \mathcal{X}_{-}=\int_{0}^{\infty} \frac{d k}{2 \sqrt{\pi k}}\left(p_{b}(k) e^{-2 i k v}+p_{b}^{\dagger}(k) e^{2 i k v}\right) .
\end{aligned}
$$

The classical quadratic constraints $(14,21)$ cannot be implemented operatorially, since as operator equations they are in contrast with the quantization rules $(34,37,38)$ and further they exhibit the usual bosonic string anomaly, $c=2$ (see e.g. [5]). However this does not concern us: in the present scheme the generators of reparametrizations are the $\mathcal{C}^{\alpha}$ and there is no anomaly for them since they commute. So we may carry out a different quantization scheme, quantizing the theory with the linear constraints (17). Then the Gupta-Bleuler procedure can be carried out following the lines of QED [17]. The vacuum is chosen as (string vacuum)

$$
a_{\alpha}\left|0>=0, \quad b_{\alpha}\right| 0>=0 .
$$

This leads to negative norm states. Now we implement the constraints by requiring that, for each oscillation mode, physical states be selected by

$$
q_{a}\left|\Psi>=0, \quad q_{b}\right| \Psi>=0 .
$$

The states $\mid\left\{n_{a}, n_{b}\right\}>$ defined as

$$
\left|\left\{n_{a}, n_{b}\right\}>\equiv q_{a}^{\dagger}\left(k_{1}\right) \ldots q_{a}^{\dagger}\left(k_{n_{a}}\right) q_{b}^{\dagger}\left(k_{1}^{\prime}\right) \ldots q_{b}^{\dagger}\left(k_{n_{b}}^{\prime}\right)\right| 0>
$$

satisfy the condition (42) (remember that $\left[q_{a, b}(k), q_{a, b}^{\dagger}\left(k^{\prime}\right)\right]=0$ ), and have zero norm if $n_{a} \neq 0$ or $n_{b} \neq 0$. The general solution of the constraints (42) is then

$$
\left|\Psi>=\sum_{n_{a}, n_{b}} \int d^{n_{a}} k \int d^{n_{b}} k^{\prime} C_{n_{a} n_{b}}\left(k_{1}, \ldots k_{n_{a}} ; k_{1}^{\prime}, \ldots k_{n_{b}}^{\prime}\right)\right|\left\{n_{a}, n_{b}\right\}>.
$$

The norm of this state is

$$
<\left.\Psi|\Psi>=| C_{00}\right|^{2}
$$

The constraints $\mathcal{H}=0, \mathcal{P}=0$ hold for matrix elements:

$$
<\Psi_{2}|: \mathcal{H}:| \Psi_{1}>=0, \quad<\Psi_{2}|: \mathcal{P}:| \Psi_{1}>=0
$$

where the normal ordering with annihilation operators on the right must be used (the algebra of $\mathcal{H}, \mathcal{P}$ shows an anomaly, but this is irrelevant here, as now our generators of gauge transformations (reparametrizations) are the constraints $\mathcal{C}_{\alpha}$ that have none). 
Using (22) and (44) the expectation value of $\rho$ is

$$
<\Psi|: \rho(u, v):| \Psi>=4 \frac{d F(u)}{d u} \frac{d G(v)}{d v},
$$

where

$$
\begin{aligned}
& F(u)=\int \frac{d k}{2 \sqrt{\pi k}}\left(C_{00}{ }^{*} C_{10}(k) e^{-2 i k u}+C_{00} C_{10}(k)^{*} e^{2 i k u}\right), \\
& G(v)=\int \frac{d k}{2 \sqrt{\pi k}}\left(C_{00}{ }^{*} C_{01}(k) e^{-2 i k v}+C_{00} C_{01}(k)^{*} e^{2 i k v}\right) .
\end{aligned}
$$

The result (48) is analogous to the classical relation (31a); we have of course

$$
F(u) G(v)=<\Psi\left|U_{0}(u) V_{0}(v)\right| \Psi>.
$$

Note that $<\Psi|: \rho(u, v):| \Psi>$ has the form

$$
<\Psi|: \rho(u, v):| \Psi>=h(a(u)+b(v)) \frac{d a(u)}{d u} \frac{d b(v)}{d v},
$$

which is the essence of classical staticity. Let us now consider the operator $M$, Eq. (16). The quantity $I$ that is the integrand in (16) classically vanishes. In the quantum case, each term in $I$ contains one of the operators $q_{a, b}$ or $q_{a, b}^{\dagger}$. Adopting a normal ordering, the matrix elements of $I$ between physical states vanish. This corresponds to the classical property. So,

$$
<\Psi_{2}|M| \Psi_{1}>=<\Psi_{2}\left|M_{0}\right| \Psi_{1}>.
$$

Further, the operator $M_{0}$ commutes with all the creation and annihilation operators of $A_{\alpha}$, since $M_{0}$ is the zero mode of the field $\varphi$. So we must characterize the vacuum by a further quantum number:

$$
M_{0}|0 ; m>=m| 0 ; m>\text {. }
$$

Equation (53) is of high interest. There are infinite vacua, differing by the eigenvalue of $M_{0}$. The only gauge invariant label of a state is $m$. This result is similar to the case of the Schwarzschild metric discussed in [11], where staticity was imposed from the beginning, reducing the problem to quantum mechanics, and states were labeled by the eigenvalues of the mass operator. Finally, the expectation value of $\varphi$ reads

$$
\begin{aligned}
<\Psi ; m|: \varphi(u, v):| \Psi ; m> & =2 m-2<\Psi ; m\left|U_{0}(u) V_{0}(v)\right| \Psi ; m> \\
& =2 m-2 F(u) G(v)
\end{aligned}
$$

in analogy to $(31 b)$.

We conclude with two remarks. The first is that the roles of $A_{0}$ and $A_{1}$ can be interchanged, i.e. the sign in Eq. (34) can be changed, because the condition (14a) 
shows that the choice of the "right" metric field is irrelevant. The operators $q_{a}$ and $q_{b}$ will again contain one operator with wrong metric and one with right metric; nothing changes in the construction (45) of the physical states. Second remark: our quantization rule (34) amounts to assuming that $x_{0}$ is time, namely that the canonical equal $x_{0}$ commutators for $A_{0}$ hold. The construction of the physical states is actually independent of which variable, $x_{0}$ or $x_{1}$, is chosen as time in defining the canonical commutators. Indeed, let us proceed by canonical equal $x_{1}$ quantization for $A_{0}$. In that case the rule (37) is suitably modified, the commutators of $b_{\alpha}$ change sign: now $b_{0}$ has wrong metric while $b_{1}$ has the correct one. Again, in $q_{b}$ there appears one operator with the right and one with the wrong metric and the construction of physical states, Eq. (45), remains unchanged.

\section{Conclusions.}

We have proposed a new quantization of the model, based on the linearization of the constraints, that may be performed because in the present case we possess further information on the theory, due to the existence of the transformation (12) from the original fields to the string ones (there is no analogous property for the string). The linearized constraints generate reparametrizations. It follows then that the Gupta-Bleuler procedure can be applied in the present case and there are no anomalies. Classically, taking into account the constraints (21), all the field theory tells us is just that there is a single free field whose degrees of freedom correspond to reparametrization of the coordinate; indeed, a choice for $U_{0}(u), V_{0}(v)$ defines $\Psi$, and the different choices correspond to different solutions (11). In the quantum theory, the physics contained in $A_{\alpha}$ is pure gauge, equivalent to free electrodynamics of longitudinal and scalar photons, and in this respect the state $|\Psi\rangle$ conveys the information correspondent to the classical case. What is physically important is the eigenvalue of the constant operator $M_{0}$, Eq. (53). The vacuum has a quantum number: the eigenvalue of the mass operator. Thus the theory is reduced essentially to quantum mechanics, while the rest is coordinate reparametrization. One may conjecture that this mechanism is at the basis of the dimensional reduction for all the quantum field models for which classically the Birkhoff theorem holds. For the general model (1) the problem is the existence and identification of the canonical transformation, analogous to (12), that leads to free fields.

\section{Appendix. Linearization of the constraints.}

The Hamiltonian and supermomentum constraints are quadratic in the canonical coordinates and momenta. We will show that we have further positivity information in the present case, that allows to linearize the constraints. It follows that the quantization of this model is not equivalent to the quantization of the bosonic string. Let us see this in detail. The constraints $\mathcal{H}_{ \pm}=0$ can be cast in the form

$$
\begin{aligned}
& \left(\pi_{0}-\lambda A_{0}^{\prime}\right)^{2}=\left(\pi^{1}+\lambda A_{1}^{\prime}\right)^{2}, \\
& \left(\pi_{0}+\lambda A_{0}^{\prime}\right)^{2}=\left(\pi^{1}-\lambda A_{1}^{\prime}\right)^{2} .
\end{aligned}
$$


Classically Eqs. (a.1) can be satisfied if one of the following cases holds:

$$
\begin{aligned}
& i \pi^{0}-\pi^{1}=0 \text { and } A_{0}^{\prime}+A_{1}^{\prime}=0 ; \\
& \text { ii } \mathcal{C}^{\alpha}=0 \text {; } \\
& \text { iii } \mathcal{D}^{\alpha}=0 \text {; } \\
& i v \pi^{0}+\pi^{1}=0 \text { and } A_{0}^{\prime}-A_{1}^{\prime}=0 .
\end{aligned}
$$

Case $i$ coincides with the choice (26). For the bosonic string all four cases are possible. In the present model the fields must satisfy the relations (12) that select $i i$ as the only option. Consider for example the case $i i i$ and use (12). This is clearly inconsistent because it implies $e^{f / 2} \cosh \Sigma=0$. Analogously, cases $i$ and $i v$ are not consistent with the canonical transformation (12). As a consequence, the linearized constraints $\mathcal{C}^{\alpha}=0$ of $i i$ are completely equivalent to the constraints $(a .1)$.

One can ask why the linearization of the constraints is so "asymmetric" and the case 2) is the only consistent choice. (Why not iii?) The answer to this question can be found in the canonical transformation (12). The connection between the twodimensional dilaton-gravity and the bosonic string was derived on the assumption that $\rho$ is positive $\left(\rho=e^{f}\right)$. This leads to the constraints $(5 a, b)$ and to Eqs. $(14 a, b)$ via the transformation (12). Let us suppose for a moment that $\rho=-e^{f}$. Since the Ricci scalar is an odd function of the metric, the first three terms of $\mathcal{H}$ and $\mathcal{P}$ have opposite signs with respect to $(5 a, b)$. (Alternatively, an overall opposite sign and $\lambda \rightarrow-\lambda$.) In this case the canonical transformation leading to the bosonic string reads

$$
\begin{aligned}
& A_{0}=-\frac{2}{\lambda} e^{-f / 2}\left(\pi_{f} \cosh \Sigma-\varphi^{\prime} \sinh \Sigma\right), \pi^{0}=\lambda e^{f / 2} \cosh \Sigma+\lambda A_{1}^{\prime}, \\
& A_{1}=-\frac{2}{\lambda} e^{-f / 2}\left(\pi_{f} \sinh \Sigma-\varphi^{\prime} \cosh \Sigma\right), \quad \pi^{1}=-\lambda e^{f / 2} \sinh \Sigma-\lambda A_{0}^{\prime} .
\end{aligned}
$$

Repeating the linearization of the constraints illustrated above it is easy to see that the "consistent linearized constraints" are now represented by the choice iii. In conclusion we can say that the choice of the linearization corresponds to the sign of the two-dimensional metric.

Let us go back to $\rho>0$ and conclude this discussion with an important remark about the signs of $\mathcal{X}_{ \pm}^{\prime}$. From the canonical transformation (12) it is straightforward to see that

$$
\begin{aligned}
& \mathcal{D}^{0}=-\lambda e^{f / 2} \cosh \Sigma, \\
& \mathcal{D}^{1}=e^{f / 2} \sinh \Sigma .
\end{aligned}
$$

As a consequence we have ( $\lambda$ is positive)

$$
\begin{aligned}
-2 \mathcal{X}_{-}^{\prime} & \equiv \frac{1}{\sqrt{\lambda}}\left(\mathcal{D}^{0}+\mathcal{D}^{1}\right)=-\sqrt{\lambda} e^{f / 2} e^{-\Sigma}<0, \\
2 \mathcal{X}_{+}^{\prime} & \equiv \frac{1}{\sqrt{\lambda}}\left(\mathcal{D}^{0}-\mathcal{D}^{1}\right)=-\sqrt{\lambda} e^{f / 2} e^{\Sigma}<0 .
\end{aligned}
$$


The definite signs of both functions $\mathcal{X}_{+}^{\prime}, \mathcal{X}_{-}^{\prime}$ allows them to be embedded into the Lagrange multipliers.

\section{Acknowledgments}

This investigation was supported in part by the grants RFBR97-01-01041 and INTAS 93-127-ext. One of us (M.C.) is supported by a Human Capital and Mobility grant of the European Union, contract number ERBFMRX-CT96-0012.

\section{References.}

[1] A. Strominger, "Les Houches Lectures on Black Holes", e-print Archive: hepth/9501071.

[2] A.T. Filippov, Mod. Phys. Lett. A11, 1691 (1996); Int. J. Mod. Phys. A12, 13 (1997).

[3] C. Callan, S. Giddings, J. Harvey and A. Strominger, Phys. Rev. D45, 1005 (1992); H. Verlinde, in Sixth Marcel Grossmann Meeting on General Relativity, M. Sato and T. Nakamura, eds. (World Scientific, Singapore, 1992).

[4] D. Cangemi and R. Jackiw, Phys. Rev. Lett. 69, 233 (1992); Phys. Rev. D50, 3913 (1994); Phys. Lett. B337, 271 (1994).

[5] E. Benedict, R. Jackiw and H.-J. Lee, Phys. Rev. D54, 6213 (1996); D. Cangemi, R. Jackiw and B. Zwiebach, Ann. Physics (N.Y.) 245, 408 (1995).

[6] K.V. Kuchař, J.D. Romano and M. Varadarajan, Phys. Rev. D55, 795 (1997) and references therein.

[7] T. Banks and M. O'Laughlin, Nucl. Phys. B362, 649 (1991).

[8] D. Louis-Martinez, J. Gegenberg and G. Kunstatter, Phys. Lett. B321, 193 (1994); D. Louis-Martinez and G. Kunstatter, Phys. Rev. D52, 3494 (1995).

[9] D. Amati, S. Elitzur and E. Rabinovici, Nucl. Phys. B418, 45 (1994).

[10] T. Strobl, Proc. of the Second Meeting on Constrained Dynamics and Quantum Gravity, Nucl. Phys. Suppl. B57, 330 (1997) .

[11] M. Cavaglià, V. de Alfaro and A.T. Filippov, Int. J. Mod. Phys. D4, 661 (1995); Int. J. Mod. Phys. D5, 227 (1996).

[12] J. Cruz and J. Navarro-Salas, Mod. Phys. Lett. A12, 2345 (1997); J. Cruz, J.M. Izquierdo, D.J. Navarro, and J. Navarro-Salas, "Free Fields via Canonical Transformations of Matter Coupled 2d Dilaton Gravity Models", Preprint FTUV-97-10, e-Print Archive: hep-th/9704168.

[13] R. Jackiw, Proc. of the Second Meeting on Constrained Dynamics and Quantum Gravity, Nucl. Phys. Suppl. B57, 160 (1997) . 
[14] K.V. Kuchař, Phys. Rev. D50, 3961 (1994).

[15] L.D. Landau and E.M. Lifshitz, The Classical Theory of Fields (Pergamon Press, 1962).

[16] W. Kummer and S.R. Lau, Ann. Phys. 258, 37 (1997).

[17] See for instance: J.M. Jauch and F. Rohrlich, The Theory of Photons and Electrons (Addison-Wesley, 1955) p. 103. 